\section{Modelado de interacciones del ADN con fármacos de actividad anti-cancerígena mediante el método ONIOM}

\author{
Modeling of DNA interactions with anti-cancer activity drugs \\ using the ONIOM method
Modelando interações de DNA com drogas anticancerígenas usando o método ONIOM

Fabiana Salazar

fabianasalazar95@gmail.com

\section{RESUMEN}

Introducción: Los complejos metálicos juegan un importante papel en la lucha contra el cáncer; estos fármacos interaccionan con el ADN mediante enlaces covalentes con las bases nitrogenadas, provocando distorsión de la hebra para su posterior reconocimiento por proteínas que inducen la muerte celular. Objetivo: Caracterizar a través de métodos computacionales basados en Mecánica Cuántica y Mecánica Molecular, la interacción entre diversos fármacos anti-cancerígenos y el ADN. Materiales y métodos: Se optimizaron todas las estructuras de dichos fármacos (complejos de $\mathrm{Pt}(\mathrm{II}), \mathrm{Ag}(\mathrm{I})$ y $\mathrm{Pd}(\mathrm{II})$ ) y las bases nitrogenadas del ADN (A, $C, G$ y $T$ ), empleando DFT al nivel B3LYP y las bases atómicas $6-31++G(d, p)$ para los átomos livianos, mientras que el dodecaedro del ADN se optimizó mediante mecánica molecular empleando UFF como campo de fuerza. Resultados: A través del análisis de los mapas de ESP y Energía de lonización Local Promedio, se determinó que el sitio más nucleofílico en las bases nitrogenadas correspondió al $\mathrm{N} 1$ de la adenina. La especie más electrofilia fue el cis-diamindiaquoplatino (II) con aumento del carácter electrofílico tras la sustitución de grupos salientes por ligandos agua. Los sistemas fármaco/base nitrogenada se caracterizaron a través de QTAIM, observándose una interacción de capa cerrada en todos los sistemas. Los sistemas de $\mathrm{Pt}$ y $\mathrm{Pd}$ con guanina resultaron ser los más estables. Conclusión: Se determinó que el método ONIOM fue capaz de modelar la torcedura que sufre la molécula de $A D N$ al coordinarse con complejos platinados, por lo tanto, para fines de observar geometrías moleculares, resultó confiable.

Palabras clave: ADN; fármacos; anticancerígenos; cáncer; molecular

\begin{abstract}
Introduction: Introduction: Metal complexes play an important role in the fight against cancer; these drugs interact with DNA through covalent bonds with nitrogenous bases, causing distortion of the strand for subsequent recognition by proteins that induce cell death. Objective: To characterize, through computational methods based on Quantum Mechanics and Molecular Mechanics, the interaction between various anti-cancer drugs and DNA. Materials and methods: All the structures of these drugs (complexes of $\mathrm{Pt}(\mathrm{II}), \mathrm{Ag}(\mathrm{I})$ and $\mathrm{Pd}(\mathrm{II})$ ) and the nitrogen bases of DNA (A, C, G and T) were optimized, using DFT at the level B3LYP and the atomic bases 6-31++ $G(d, p)$ for light atoms, while the DNA dodecahedron was optimized by molecular mechanics using UFF as a force field. Results: Through the analysis of the ESP and Average Local lonization Energy maps, it was determined that the most nucleophilic site in the nitrogenous bases corresponded to the N1 of the adenine. The most electrophilia species was cis-diamindiaquoplatin (II) with an increase in electrophilic character after substitution of leaving groups with water ligands. Nitrogenous drug / base systems were characterized through QTAIM, with a closed layer interaction observed in all systems. The $\mathrm{Pt}$ and $\mathrm{Pd}$ systems with guanine proved to be the most stable. Conclusion: It was determined that the ONIOM method was able to model the twist that the DNA molecule undergoes when coordinating with platinum complexes, therefore, for the purpose of observing molecular geometries, it was reliable.
\end{abstract}

FS: Universidad de Carabobo/ Instituto Venezolano de Investigaciones Científicas (IVIC), Venezuela.

Key words: DNA; drugs; anticancer; cancer; molecular 
FS: Universidad de Carabobo/ Instituto Venezolano de Investigaciones Científicas (IVIC), Venezuela.

\begin{abstract}
RESUMO
Introdução: Complexos metálicos desempenham um papel importante na luta contra o câncer; essas drogas interagem com o DNA através de ligações covalentes com as bases de nitrogênio, causando distorção do fio para posterior reconhecimento por proteínas que induzem a morte celular. Objetivo: Caracterizar através de métodos computacionais baseados em Mecânica Quântica e Mecânica Molecular, a interação entre várias drogas anticancerígenas e DNA. Materiais e métodos: Otimizado todas as estruturas dessas drogas (Pt(II), complexos $\mathrm{Ag}(\mathrm{I})$ e Pd(II) e bases de nitrogênio de DNA (A, C, G e T), usando DFT a nível b3LYP e bases atômicas 6-31++G(d,p) para átomos de luz, enquanto o dodecaedro de DNA foi otimizado pela mecânica molecular usando uff como campo de força. Resultados: Através da análise dos mapas esp e energia da ionização local média, foi determinado que o local mais nucleílico dos bases de nitrogênio correspondiam ao N1 da adenina. A espécie mais eletrofilalfoi cis-diamindiaquoplatin (II) com maior caráter eletrofilico após substituição de grupos de saída por ligas de água. Os sistemas de nitrogênio droga/base foram caracterizados através da QTAIM, com uma interação de camada fechada observada em todos os sistemas. Os sistemas pt e PD com guanine acabaram por ser os mais estáveis. Conclusão: Foi determinado que o método ONIOM foi capaz de modelar a torção da molécula de DNA coordenando com complexos banhados a platina, portanto, com o propósito de observar geometrias moleculares, mostrou-se confiável.
\end{abstract}

Palavras-chave: DNA; drogas; anticâncer; câncer; molecular

\section{INTRODUCCIÓN}

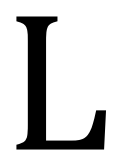
os métodos basados en Mecánica Cuántica $(\mathrm{QM}$ por sus siglas en inglés) definen el estado de un sistema a partir de la ecuación de Schrödinger, permitiendo aproximar la energía de una molécula con un tratamiento explícito de su estructura electrónica. Esto implica un alto costo computacional (en términos de consumo de tiempo y memoria) asociado al cálculo de términos matemáticos que cuantifican la interacción de los electrones, proporcionando una descripción precisa del comportamiento de los mismos y por lo tanto, de la estructura de los sistemas químicos (1).

Debido a que el costo computacional resulta ser relativamente bajo en comparación con los métodos mecanocuánticos, es posible con los computadores que se disponen en la actualidad modelar sistemas de gran tamaño (de hasta $10^{4}$ átomos). Como herramienta, la química computacional puede ser aplicable a otras áreas como la física, la biología y la bioquímica. Una aplicación específica de la química computacional ha sido en el modelaje de las interacciones y el comportamiento de los sistemas biológicos (2).

Por si solos, los métodos MM y QM parecen ser exiguos al modelarlos y esta carencia llevó a pensar en nuevos métodos computacionales. De esta premisa surgen los métodos híbridos (entre ellos el método ONIOM) capaces de aprovechar las ventajas de la mecánica cuántica y la mecánica molecular (3). Este método permite estudiar los sistemas complejos al fragmentarlos en dos o más regiones a los cuales se les aplican diferentes niveles de teoría. Un ejemplo de ello es el estudio de las interacciones de los ácidos nucleicos con complejos metálicos, donde a la región del sitio químicamente activo, se aplican métodos $a b$ initio y en el resto del sistema métodos de mecánica molecular $(3,4)$.

En consecuencia a lo expuesto, se plantea estudiar las propiedades electrónicas y termodinámicas de fármacos anticancerígenos, con el objetivo de modelar las interacciones entre estos y el ADN y analizar la influencia de los 
complejos metálicos en la estructura helicoidal.

El cáncer es un grupo de enfermedades caracterizadas por el crecimiento celular no regulado y la invasión y diseminación de células desde el sitio de origen a otros sitios en el cuerpo (metástasis) (5). Es una enfermedad genética, es decir, es causada por cambios en los genes que controlan el funcionamiento de las células, específicamente aquellos que controlan el crecimiento y la división. Los cambios pueden heredarse o adquirirse durante la vida de una persona, ya sea como resultado de errores en el ADN (Ácido desoxirribonucleico) o por exposición a sustancias carcinógenas. Algunos cambios afectan solo una unidad de ADN, lo que se llama un nucleótido, mientras que otros cambios comprenden tramos más grandes (6).

Entre las terapias anti-cancerígenas implementadas con mayor frecuencia se encuentra la quimioterapia, que debe su nombre al uso de sustancias químicas para tal fin. Estas sustancias suelen ser fármacos a base de complejos de metales de transición, mayormente complejos platino (Pt) como el cisdiamindicloroplatino (II) (7). El cisdiamindicloroplatino (II) (o cisplatino como comúnmente se le conoce), es un complejo de platino (II) con geometría plano cuadrada y cuyos ligandos $(-\mathrm{Cl}$ y $-\mathrm{NH}_{3}$ ) se encuentran en posición cis. Su uso clínico generalizado ha provocado el aumento de su toxicidad y resistencia en el organismo, por lo que hoy en día solo se usa en combinación con otros compuestos (7). Estos complejos de platino tienen como blanco de acción al ADN, con el cual interaccionan covalentemente a través del N7 de la guanina y formando productos conocidos como aductos (7). Así, las interacciones entre el ADN y los complejos de platino (II) son objeto de investigaciones que buscan comprender el efecto que estos fármacos ejercen sobre la estructura del ADN (8). En este sentido, la química computacional se ha convertido en una herramienta útil para su estudio, permitiendo el modelado y análisis de estos sistemas, y la comprensión de las interacciones moleculares que tienen lugar en los mismos (1).

Esto conlleva a tratar el sistema ADN/fármaco con un método multiescala, es decir, un método que combine una descripción cuántica (QM) en la región de interés (donde el fármaco se enlaza a las bases del ADN) con una descripción clásica (MM) en el resto del entorno (1). A este tratamiento se le conoce como método QM: MM (en este caso particular ONIOM) y se ha evidenciado un aumento en su uso para estudiar los procesos biológicos (4).

El incremento del cáncer en la población mundial ha llevado a organizaciones como la Organización Mundial de la Salud (OMS) al desarrollo de programas informativos sobre las causas, riesgos, prevención y tratamiento de esta enfermedad. Una de las medidas para el control del cáncer está enfocada a comprender el mecanismo de acción de los fármacos (6). El blanco de acción es diverso, pueden actuar sobre el ADN rompiendo la hélice, interfiriendo con las proteínas relacionadas con el ADN o modificando la expresión de genes específicos (9). Los complejos de Platino (II), por ejemplo, actúan directamente sobre la hélice del ADN provocando su distorsión y posteriormente la apoptosis (9). Este mecanismo de acción se ha convertido un punto clave para comprender la actividad biológica de los complejos metálicos (8).

El modelaje de los fármacos y sus interacciones mediante técnicas computacionales permite dilucidar 
propiedades moleculares (distribución de la densidad electrónica, energía de ionización, basicidad y acidez, nucleofilicidad) (9) y termodinámicas responsables de la afinidad global y especificidad de los mismos (9). Estudios combinados pueden realizarse a través del método ONIOM, el cual fragmenta el sistema en varias regiones a fin de estudiar cada región mediante distintos niveles de teoría (1). Las interacciones de los ácidos nucleicos con complejos metálicos han sido estudiadas y aplicadas exitosamente mediante el método ONIOM (9), por lo que se hará uso del mismo para estudiar las interacciones del ADN con complejos de Pt (II).

\section{MATERIALES Y MÉTODOS}

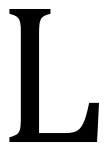
a investigación se llevó a cabo empleando paquetes de software libre para el cálculo de propiedades moleculares y la construcción y visualización de las geometrías moleculares. Los cálculos ONIOM, QM y MM se realizaron mediante el programa Gaussian. Para la construcción de los modelos moleculares se usó el programa Avogadro versión 1.2.0, el cual es un editor y visualizador de moléculas avanzado diseñado para el uso multiplataforma en química computacional (10).

\section{Sistema de estudio}

El estudio se realizó sobre distintos fármacos anti-cancerígenos, las bases nitrogenadas del ADN (A, C, G y T), sistemas fármaco/base nitrogenada y el sistema ADN/fármaco. Los fármacos modelados fueron tres complejos de Pt (II) (cisplatino, carboplatino y oxaliplatino) y dos (2) complejos metal-NHC de Pd (II) y Ag (I) respectivamente. La hebra de ADN presentó un total de doce nucleótidos (dodecaedro) en la secuencia d (CCTCTGGTCTCC) y los fármacos de interés interaccionaron con ésta mediante el N7 de la guanina a través de enlaces intracadena (al ser estos los de mayor interés biológico). La secuencia de ADN empleada se tomó de un estudio RMN realizado por Gelaso y Lippard (11). Donde determinaron la estructura de la macromolécula al interaccionar con el cisdiaminplatino (II) a través de un enlace intracadena d (GpG). Los sistemas ADN/fármaco se estudiaron independientemente uno del otro mediante el método ONIOM (QM: MM) mientras que los sistemas fármaco/base nitrogenada se estudiaron a través cálculos mecano-cuántica a nivel DFT.

\section{Archivos de entrada en Gaussian}

Las estructuras moleculares de los sistemas de estudio construidas se ejecutaron con el programa GaussView versión 5.0. El cuadro de diálogo Gaussian Calculación Setup (Configuración de cálculos de Gaussian) proporciona acceso a todas las principales características de Gaussian (12).

\section{Cálculos mecano-cuánticos (QM): optimización de geometría}

Gaussian09 permite realizar cálculos mecano-cuántica a diversos niveles de teoría. Las funciones de polarización se eligieron para modelar la distribución electrónica entre átomos enlazados y las funciones difusas para considerar la densidad electrónica lejos de los núcleos que poseen pares solitarios de electrones (nitrógeno, fósforo y oxígeno) (13).

Finalmente se especifica el nombre del archivo wfn que se quiere generar. Gen permite especificar un conjunto de bases dado por el usuario (14). Opt permite que se realice una optimización geométrica. El algoritmo predeterminado es el Algoritmo de Berny usando gediis. MaxCycle 
establece el número máximo de pasos de optimización (15). Input hace que el conjunto de base actual se imprima en una forma adecuada para usar como entrada de conjunto de base general y, por lo tanto, puede usarse para agregar o modificar conjuntos de bases estándar (16).

\section{Cálculos de mecánica molecular (MM): optimización de la geometría del ADN}

La estructura molecular del ADN se exportó desde Avogadro en formato .mol para ser leído con GaussView y se optimizó mediante el módulo de mecánica molecular de Gaussian09 utilizando UFF como campo de fuerza. Uff indica uno de los tres campos de fuerza que Gaussian tiene disponible para cálculos de MM y ONIOM (17).

\section{Cálculo ONIOM (QM: MM): optimización de la geometría del sistema ADN fármaco}

Las geometrías optimizadas de los fármacos y el $\mathrm{ADN}$ se usaron para modelar los sistemas ADN/fármaco, en los cuales el centro metálico se unió covalententemente al ADN mediante un enlace d (GpG). Las geometrías se optimizaron a través del método ONIOM.

\section{Cálculo de propiedades moleculares}

El cálculo del Potencial Electroestático (ESP) y la Energía de Ionización Local Promedio se realizó mediante el programa
Multiwfn versión 3.4.1 (18). Multiwfn realiza análisis de funciones de onda electrónicas de manera clara. El archivo .wfn se ejecutó generando dos archivos para cada propiedad: .xyz y .cub, y mediante el programa wfn to binary (19).

\section{Cálculo QTAIM}

La caracterización computacional de las interacciones entre los fármacos y sistemas, fármaco/base nitrogenada se realizó mediante análisis topológico de la densidad electrónica a través del programa $A I M-U C$ versión 1.6.4 (20). AIM-UC es un programa que realiza algunos gráficos relacionados con QTAIM de Richard Bader a partir de un mallado 3D de densidad de carga electrónica (20).

\section{RESULTADOS}

A través del análisis de los mapas de ESP y Energía de Ionización Local 1 Promedio, se determinó que el sitio más nucleofílico en las bases nitrogenadas correspondió al N1 de la adenina. La especie más electrofilia fue el cis-diamindiaquoplatino (II) con aumento del carácter electrofílico tras la sustitución de grupos salientes por ligandos agua. Los sistemas fármaco/base nitrogenada se caracterizaron a través de QTAIM, observándose una interacción de capa cerrada en todos los sistemas. Los sistemas de Pt y Pd con guanina resultaron ser los más estables. Se detalla a continuación: 
Tabla 1. Distancias y ángulos de enlace alrededor del centro metálico de los diferentes sistemas ADN/cisplatino

\begin{tabular}{|c|c|c|c|c|c|c|c|}
\hline & ADN 1 & ADN 2 & ADN 3 & Exp. & Desv. $1^{a}$ & Desv. 2 & Desv. 3 \\
\hline Pt-N7 ${ }^{a}\left({ }^{\circ} \mathrm{A}\right)$ & 2,092 & 2,089 & 2,076 & 2,051 & 2,0 & 1,9 & 1,2 \\
\hline $\mathrm{Pt}^{-N 7^{b}}\left({ }^{\circ} \mathrm{A}\right)$ & 2,069 & 2,059 & 2,052 & 2,046 & 1,1 & 0,6 & 0,3 \\
\hline Pt-N1 $1^{A m}\left({ }^{\circ} \mathrm{A}\right)$ & 2,080 & 2,090 & 2,100 & 2,050 & 1,5 & 2,0 & 2,4 \\
\hline Pt-N2 ${ }^{A m}\left({ }^{\circ} \mathrm{A}\right)$ & 2,088 & 2,089 & 2,102 & 2,049 & 1,9 & 2,0 & 2,6 \\
\hline $\mathrm{N} 7^{a}-\mathrm{Pt}-\mathrm{N} 7^{b}\left(^{0}\right)$ & 92,2 & 90,3 & 86,8 & 90,1 & 2,3 & 0,2 & 3.7 \\
\hline \multirow[t]{2}{*}{$\begin{array}{l}\mathrm{N} 1^{A m_{-} \mathrm{Pt}-\mathrm{N} 2^{A m}} \\
\left(^{A}\right)\end{array}$} & 93,3 & 94,8 & 98,5 & 90,1 & 3,6 & 5,2 & 9,3 \\
\hline & & & & $\mathbf{M A P E}^{b}$ & 2,1 & 2,0 & 3,3 \\
\hline
\end{tabular}

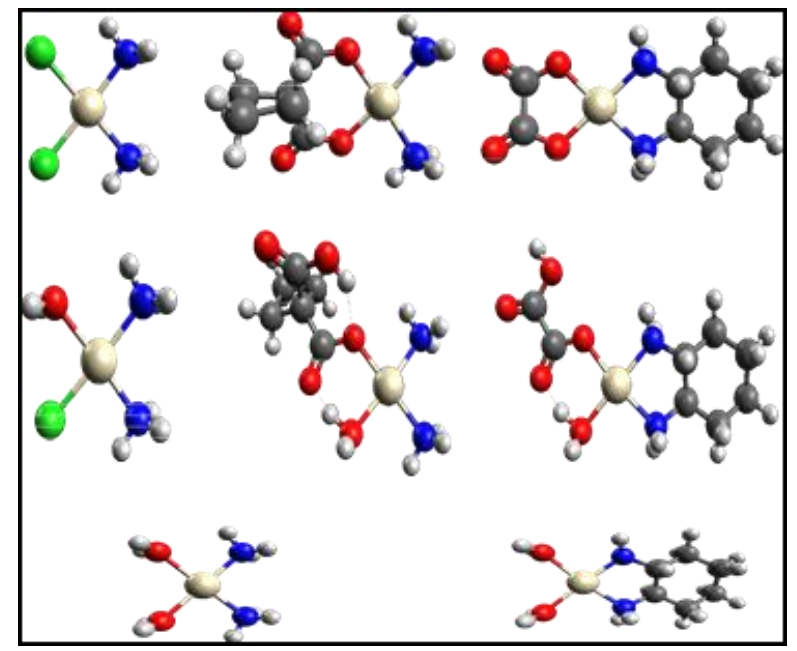

Figura 1. Geometrías optimizadas de los complejos de Pt (II). De arriba a abajo y de izquierda a derecha: Cispt, Carbopt, Oxalipt, Monoaquocispt, Monoaquocarbopt, Monoaquooxalipt, Diaquocispt, Diaquooxalipt.

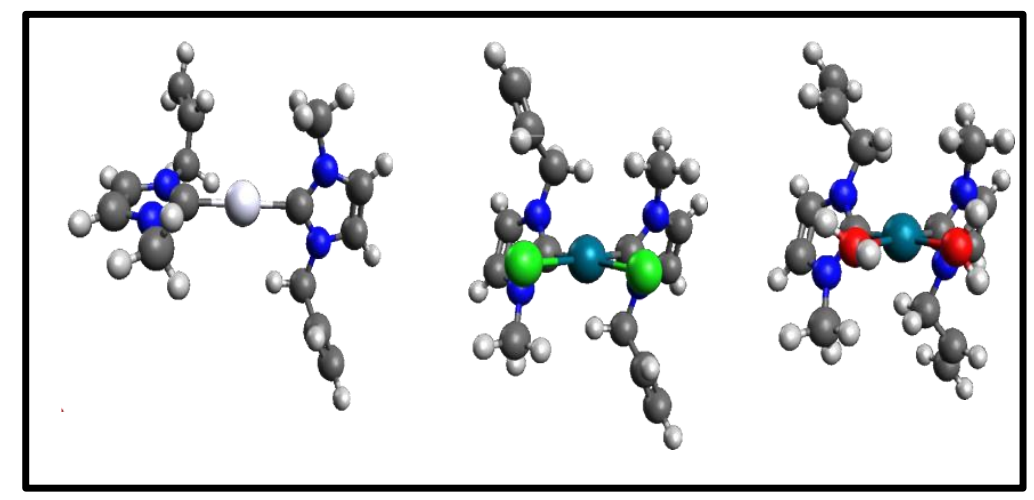

Figura 2. Geometrías optimizadas de los complejos de Ag (I) y Pd (II). De izquierda a derecha: AgCarb, Pd-Carb-Cl y Pd-Carb- $\mathrm{H}_{2} \mathrm{O}$. 


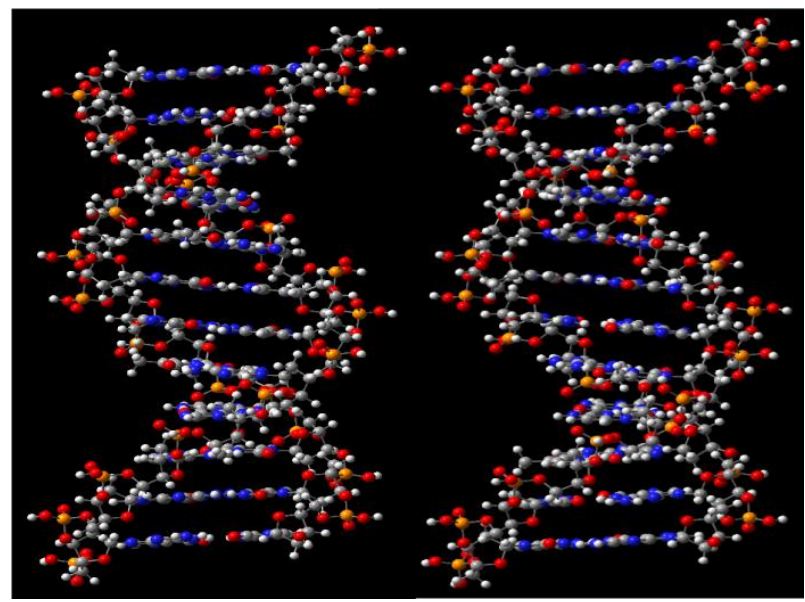

Figura 3. Dodecámero de ADN. A la izquierda se muestra el sistema antes de realizar la optimización de geometría mediante mecánica molecular. A la derecha se muestra la estructura optimizada. No se observaron cambios estructurales.

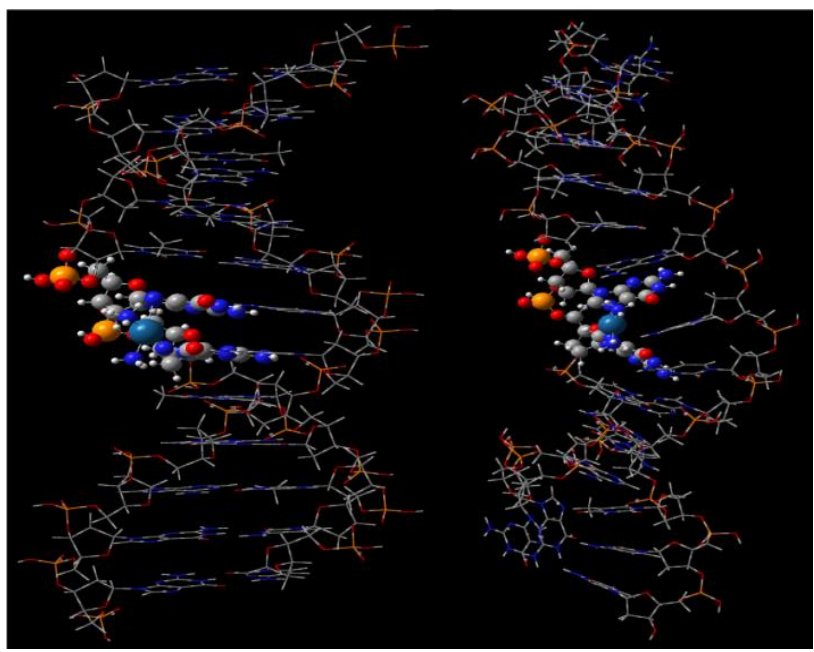

Figura 4. Sistema ADN/Cisplatino (ADN 2). A la izquierda se muestra el sistema antes de realizar la optimización de geometría mediante el método ONIOM. A la derecha se muestra la estructura optimizada. Total, de átomos en la región QM: 79. Total de átomos en la región MM: 720. Total, de átomos en todo el sistema: 799 .

\section{DISCUSIÓN}

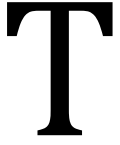

odas las estructuras moleculares fueron optimizadas a través del programa Gaussian09 y mediante un análisis de frecuencia, se confirmó que las mismas correspondían a un mínimo en la superficie de energía potencial al no poseer frecuencias de vibración imaginarias. Asimismo, el mecanismo de acción de los fármacos anti-cancerígenos (basados en complejos de Pt) implica la interacción covalente con las bases del ADN. El ESP tiene mayor aplicabilidad en las reacciones que implican interacciones electrostáticas reconociendo las zonas de mayor y menor concentración electrónica, mientras que la energía de ionización local promedio es más eficiente en las reacciones que implican transferencia de carga o interacciones de índole covalente. 


\section{CONCLUSIONES}

$\mathrm{L}$ os electrones más polarizable en las bases nitrogenadas correspondieron al: N3 en la citosina, N1 en la adenina, N7 en la guanina y C5 en la timina, mientras que los sitios de mayor concentración electrónica correspondieron al: 08 en la timina y N3 en la adenina (A).

En las bases nitrogenadas, el ESP no identificó los sitios de ataque electrofílico que se mencionan comúnmente en la literatura, resultado que si observó con la energía de ionización local promedio. Además, a partir de $\Gamma(\mathbf{r})$ se identificó a la adenina como la purina con mayor carácter nucleofílico, mientras que en las pirimidina resultó ser la citosina. Los grupos aceptores de electrones (como el grupo carbonilo) disminuyen la basicidad de la amina aromática al deslocalizar el par electrónico solitario del nitrógeno en el sistema $\pi$ del anillo. De acuerdo a lo anterior, la función $I(\mathbf{r})$ fue más precisa que el ESP al identificar los sitios más propensos para la formación del enlace fármaco/base nitrogenada en los aductos con el ADN (20).

En los complejos metálicos, los electrones más polarizable se ubicaron en el centro metálico para los complejos de $\mathrm{Pt}$ (II) y $\mathrm{Ag}$ (I), entre los átomos de $\mathrm{Cl}$ en el $\mathrm{Pd}$-Carb-Cl y en el enlace doble $\mathrm{C}$-C de la estructura del propileno del Carbeno heterocíclico en el Pd-Carb- $\mathrm{H}_{2} \mathrm{O}$. El carácter polarizable de estos electrones decrece en el siguiente orden: Oxalipt > cispt $>$ Pd-Carb-Cl $>$ carbopt $>$ AgCarbmonoaquooxalipt $>$ Monoaquocispt $>$ monoaquocarbopt $>$ Pd-Carb- $\mathrm{H}_{2} \mathrm{O}>$ diaquooxalipt $>$ diaquocispt.

Por su parte, las regiones de mayor concentración electrónica en los fármacos correspondieron a los grupos salientes en los complejos de Pt (II) y el Pd-carb-Cl, y al enlace doble $\mathrm{C}-\mathrm{C}$ de la estructura del propileno del Carbeno heterocíclico en los complejos Ag-Carb y Pd-Carb- $\mathrm{H}_{2} \mathrm{O}$. Se observó que la tendencia a aceptar electrones en estas moléculas decrece en el siguiente orden: Oxalipt > Pd-CarbCl > carbopt $>$ cispt $>$ monoaquooxalipt $>$ monoaquocarbopt $>$ Ag-Carb $>$ Pd-Carb$\mathrm{H}_{2} \mathrm{O}>$ diaquooxalipt $>$ diaquocispt.

\section{REFERENCIAS}

1. Arrebol A. Métodos de simulación QM: MM y de mecánica estadística: una aproximación teórica a la biología molecular. An. Quima. 2013; 109(4):268-275

2. Robertazzi A and Platts, J. QM: MM study of Cisplatin-DNA oligonucleotides: From simple models to realistic systems. Chem. Eur. J. 2006; 12: 5747-5756

3. Pavelka M y Burda J. Pt-bridges in various single-strand and double-helix DNA sequences. DFT and MP2 study of the cisplatin coordination with guanine, adenine, and cytosine. J Mol Model, 2007; 13: 367379

4. Petrovic M y Todorovic D. Biochemical and molecular mechanisms of action of cisplatin in cancer cells. Medicine and Biology, 2015; 18(1):12-18

5. Muggia F. Platinum Compounds: The culmination of the era of cancer chemotherapy. En: Bonetti A, Leone R, Muggia $F$ y Howell. Platinum and other heavy metal compounds in cancer chemotherapy. Roma: Springuer Nature; 2009

6. Barzaghi M. PAMoC (Versión 2002.0), Online User's Manual; CNR-ISTM, Institute of Molecular Science and Tech-nologies. Roma, 2002. Disponible en: http://www.istm.cnr.it/ barz/pamoc/

7. Gkionis K, Mutter $S$ y Platts J. Qm: mm description of platinum-dna interactions: comparison of binding and dna distortion of five drugs. RSC Adv. 2013: 4066-4073

8. López J, Ensuncho A y Robles J. Estudio teórico de la reactividad química y biológica de cisplatino y algunos derivados con actividad anticancerosa. Información tecnológica, 24(3), 3-14 doi: https://dx.doi.org/10.4067/S071807642013000300002

9. Lu FJ. Multiwfn: a multifunctional wavefunction analyzer. Comput Chem. 2012.15;33(5):580-92. doi: $10.1002 / j c c .22885$

10. Vega D. Productos de programación en lenguaje C relacionados con QTAIM. Universidad de Carabobo. 2014. Disponible en:

http://alfa.facyt.uc.edu.ve/quimicomp/aim_uc/ manual/ manual_sp.html, 2017 
11. Gaussian 09 User's reference, 2011: [citado 201715 abril ] Disponible en: http://www.gaussian.com/g_tech/g_ur/u_cub egen.htm

12. Jensen. Introduction computational chemistry. Londres: John Wiley \& Sons; 2001

13. Vega D, Aray $Y$, Rodríguez J. Comp Chem 2012;33: 2526-2531

14. Hay J, Wats W. Ab initio effective core potentials for molecular calculations. Potentials for the transition metal atoms Sc to Hg J. Chem. Phys.1985; 82(1): 270. Doi: https://doi.org/10.1063/1.448799

15. Popelier P. Atoms in molecules an introduction. Londres: Prentice Hall; 2000
16. Richard F y Bader W. Atoms in molecules: A quantum theory. New York: Oxford science publications; 1994

17. Politzer P, Murray J. The average local ionization energy: concepts. Amsterdan: Elsevier; 2008

18. Gaussian Inc. Using gaussview 6. En: Expanding the limits of computational chemistry. 2017 [citado 3 de enero 2018] Disponible en: http://gaussian.com/gv6main/

19. Gaussian Inc. Link 0 commands, En: Expanding the limits of computational chemistry. 2018 [citado 3 de enero 2018] Disponible en: http://gaussian.com/link0/

20. Gaussian Inc. Optimization. En: Expanding the limits of computational chemistry, 2018 [citado 3 de enero 2018] Disponible en: http://gaussian.com/opt/

- La autora declara no poseer conflicto de interés.

- Para la realización del estudio no se contó con ente financiador.

- Investigación realizada bajo las más estrictas normas bioéticas. 